\title{
Amiodarone and Magnesium Sulphate in Arrhythmia Prophylaxis in Pediatric Congenital Heart Diseases Patients Undergoing Cardiopulmonary Bypass
}

\author{
Azza Ali Mohamed Ali Khalil ${ }^{1}$, Al Shaymaa Ahmed Ali ${ }^{1}$, \\ Ali Mohamed Refat Ali Soliman' ${ }^{2}$ Ehab Idris Almahdoy ${ }^{* 3}$
}

Departments of ${ }^{1}$ Pediatrics and ${ }^{2}$ Cardiothoracic Surgery, Faculty of Medicine, Zagazig University, Egypt.

Department of ${ }^{3}$ Pediatrics, Faculty of Medicine - Tripoli University, Libya

*Corresponding Author: Ehab Idris Almahdoy, Mobile: +201554239501, Email: ehab.edris.mh@gmail.com

\begin{abstract}
Background: Postoperative arrhythmias are an important cause of morbidity and mortality after cardiac surgery for congenital heart disease. In the early postoperative period, patients with congenital heart disease are especially vulnerable to rhythm disturbances. Objective: This study aimed to assess safety and efficacy of prophylactic amiodarone and magnesium sulphate in preventing cardiac arrhythmia especially junctional ectopic tachycardia (JET) in children with congenital heart disease (CHD) who undergo open heart surgery. Patients and Methods: This was a comparative clinical trial that was conducted on 48 cases at Cardiothoracic Department and Pediatric Cardiology Unit, Zagazig University Hospitals. The cases were divided into three groups according to received drug; Group (A): patients receiving placebo, Group (B): patients receiving magnesium sulfate and Group (C): patients receiving amiodarone. Results: There were highly statistically significant differences between groups as regard to Inotrope Score intraoperatively, heart rate and median arterial pressure postoperatively, postoperative Inotrope Score and drug complications where $\mathrm{P}<0.001$. Conclusions: prophylactic of either amiodarone or magnesium sulfate are safe and relatively effective in preventing early postoperative arrhythmia in children after corrective heart surgery for CHD.
\end{abstract}

Key words: Amiodarone, Cardiopulmonary, Magnesium sulphate, Pediatric.

\section{INTRODUCTION}

Postoperative arrhythmias are an important cause of morbidity and mortality after cardiac surgery for congenital heart disease. In the early postoperative period, patients with congenital heart disease are especially vulnerable to rhythm disturbances ${ }^{(\mathbf{1})}$. In pediatric cardiac surgery, there is little information regarding the incidence, risk factors, management, and prophylaxis for early postoperative $\operatorname{arrhythmias}^{(2)}$. Postoperative junctional ectopic tachycardia (JET) remains one of the most common arrhythmias (8\%-20\%) after pediatric cardiac surgery. JET is associated with hemodynamic instability, longer mechanical ventilation time, and longer stays in the cardiac intensive care unit (CICU) ${ }^{(3)}$.

Although the etiology of JET is multifactorial, some studies have associated the presence of JET with hypomagnesemia during cardiopulmonary bypass (CPB) ${ }^{(4)}$. Magnesium is an important regulator of multiple cardiovascular processes, including myocardial conduction and contractility, transmembrane calcium flux, potassium transport, vascular smooth muscle tone, coronary reactivity, and nitric oxide synthesis ${ }^{(5)}$. Inasmuch as magnesium is an essential cofactor for the maintenance of myocardial transmembrane potential, magnesium deficiency decreases the threshold for arrhythmias ${ }^{(\boldsymbol{6})}$.

In adults having cardiac surgery, hypomagnesemia has been associated with the presence of arrhythmias, especially postoperative atrial fibrillation. Furthermore, in adults the administration of magnesium sulfate $\left(\mathrm{MgSO}_{4}\right)$ during cardiac surgery reduces the occurrence of postoperative atrial fibrillation ${ }^{(7)}$. In pediatric patients undergoing cardiac surgery with $\mathrm{CPB}$, hypomagnesemia is common (34\%) and is associated with longer CICU stay, higher Pediatric Risk of Mortality (PRISM) score, and longer mechanical ventilation time ${ }^{(\mathbf{8})}$.

Although magnesium supplementation during $\mathrm{CPB}$ reduces the incidence of arrhythmias in adults, the benefits of magnesium administration in pediatric cardiac patients have not been demonstrated nor have dosage guidelines been established ${ }^{(\mathbf{9})}$. Also, now, the first line of treatment of JET is intravenous amiodarone and hypothermia $^{(2)}$. However, amiodarone, which is an iodine-rich drug with strong structural similarities to the thyroid hormones, has been proved to be effective and safe in preventing postoperative $\mathrm{AF}^{(10-}$ 12).

This study aimed to assess safety and efficacy of prophylactic amiodarone and magnesium sulphate in preventing cardiac arrhythmia especially JET in children with CHD who undergoing open heart surgery.

\section{PATIENTS AND METHODS}

This was a comparative clinical trial that was conducted on 48 cases at Cardiothoracic Department and Pediatric Cardiology Unit, Zagazig University 
Hospitals through the period from October 2019 up to April 2020.

\section{Ethical approval:}

An approval of the study was obtained from Zagazig University academic and ethical committee Hospitals. Written informed consent obtained from the parents of all the participants.

Inclusion criteria: Patients who were undergoing open heart surgery for congenital heart disease at Cardiothoracic Department and Pediatric Cardiology Unit, Zagazig University. Patients ages between (2 17 years).

Exclusion criteria: Patients who were having arrhythmias or had a history of arrhythmias before surgery. Patients with marked sinus bradycardia while awake. Patients with congestive heart failure. Patients with persistent hypotension. Patients with AV block. Patients with prolonged corrected QT interval. Patients with peripheral neuropathy. Patients with clinical hypo- or hyperthyroidism. Patients with liver disease. Patients with incomplete or absent medical records. Patients who underwent emergency operations. In case of amiodarone use, we excluded patients with known history of amiodarone hypersensitivity.

Sample size: A comprehensive sample was taken including all cases with congenital heart disease undergoing open heart surgery during period of 6 months ( 8 cases /month) so, sample was equal 48 cases.

The cases were divided into three groups according to the prophylactic drug received drug; Group (I): Patients who were given placebo. 2-17 years old, ( 8 male, 8 female), Group (II): Patients who were given prophylactic magnesium sulfate. 217 years old, (8 male, 8 female), Group (III): patients who were given prophylactic amiodarone. 2-17 years old, ( 7 male, 9 female).

All patients was subjected to full history, cardiac examination included general appearance of child. General examination of systems and associated medical conditions. Heart rate, respiratory rate, temperature, blood pressure, inspection, palpation, percussion and auscultation. To detect any complication; abdominal examination for organomegaly and ascites. Anthropometric measures, which included weight, height, head circumference, mid upper arm circumference. BMI Echocardiography examination for accurate diagnosis of underlying cardiac disease. Laboratory investigation including; CBC, CRP, Kidney function tests, Liver function tests, electrolytes, thyroid function test, bleeding time.

\section{Interventional design:}

Each patient had been randomly assigned to one of the following groups in a double blinded way; Group 1: Receive placebo (5\% aqueous dextrose solution or normal saline), Group 2: Receive 25 $\mathrm{mg} / \mathrm{kg}$ of $\mathrm{MgSO}_{4}$. Single bolus of magnesium sulfate $(25 \mathrm{mg} / \mathrm{kg})$ into the CPB circuit at the beginning of rewarming. The cardioplegia solution (Baxter, Deerfield, IL, USA), which contains $0.325 \%$ of magnesium chloride, was given to all the patients as needed according to our standard CPB protocol ${ }^{(13)}$. Group 3: Receive 5 milligrams/kilogram of amiodarone had been administered intravenously over $30 \mathrm{~min}$ at the time of anesthesia induction followed by continuous infusion of $10-15 \mu \mathrm{g} / \mathrm{kg} / \mathrm{min}$ IV diluted in dextrose $5 \%$ over $24 \mathrm{~h}$ for the three postoperative days ${ }^{(2)}$.

The study medication had been prepared by the hospital pharmacy. Standard 12-lead ECGs had been registered in all patients pre and postoperative. Anesthesia had been induced. The composition of the bypass circuit and perfusion protocols had been performed according to established institutional practices.

\section{Aristotle Score}

The Aristotle scoring system was used to assess the complexity level of surgical procedures. The Aristotle basic score is a procedure-adjusted complexity score and only applies to procedures. It is based on the potential for mortality, the potential for morbidity, and the anticipated technical difficulty. The complexity was based on procedures as defined by the Society of Thoracic Surgeons and the European Association of Cardio-Thoracic Surgery International Nomenclature. Four levels of procedural complexity were defined, which matche the basic score range from 1.5 to 15 : level 1 (1.55.9), level 2 (6.0-7.9), level 3 (8-9.9), and level 4 (10.0-15.0). The level of complexity was obtained from our Children's National Medical Center Cardio access Surgical Database, which automatically calculates the basic score of the primary procedure.

\section{Follow up:}

Postoperative ECG monitoring had been in the cardiac care unit (CCU) for 3 days postoperatively. The patient's temperature, heart rate, and mean blood pressure had been recorded postoperatively

\section{Statistical Analysis}

Recorded data were analyzed using the statistical package for social sciences, version 20.0 (SPSS Inc., Chicago, Illinois, USA). Quantitative data 
were expressed as mean \pm standard deviation (SD). Qualitative data were expressed as frequency and percentage. Independent-samples t-test of significance was used when comparing between two means. Chi-square $\left(\mathrm{x}^{2}\right)$ test of significance was used in order to compare proportions between two qualitative parameters.
The confidence interval was set to $95 \%$ and the margin of error accepted was set to 5\%. P-value $<0.05$ was considered significant.

\section{RESULTS}

Supraventricular tachycardia (SVT) was the most common type of arrhythmia followed by JET, then atrial flutter (Table 1).

Table (1): Rates of postoperative and intraoperative arrhythmia in all patient

\begin{tabular}{|l|c|c|}
\hline \multicolumn{1}{|c|}{ Type of Arrhythmia } & No $=48$ & $\%$ \\
\hline \hline Supraventricular tachycardia (SVT) & 4 & 8.33 \\
\hline JET & 3 & 6.25 \\
\hline Atrial flutter & 1 & 2.083 \\
\hline Total & 8 & 16.67 \\
\hline
\end{tabular}

There were no statistically significant differences between groups as regard different types of arrhythmia. Patients on amiodarone (group C) did not have any arrhythmias (Table 2).

Table (2): Arrhythmia in the studied groups

\begin{tabular}{||l|l|l|l|l|l|l|l||}
\hline \multirow{2}{*}{ Arrhythmia } & \multicolumn{2}{|c|}{$\begin{array}{l}\text { Group (A) } \\
\text { (n=16) }\end{array}$} & \multicolumn{2}{c|}{$\begin{array}{l}\text { Group (B) } \\
\text { (n=16) }\end{array}$} & \multicolumn{2}{|c|}{$\begin{array}{l}\text { Group (C) } \\
\text { (n=16) }\end{array}$} & \multirow{2}{*}{ P } \\
\cline { 2 - 8 } & No. & \% & No. & \% & No. & \% & \\
\hline $\begin{array}{l}\text { Supraventricular } \\
\text { tachycardia (SVT) }\end{array}$ & 3 & 18.8 & 1 & 6.3 & 0 & 0 & 0.148 \\
\hline JET & 2 & 12.5 & 1 & 6.3 & 0 & 0 & 0.344 \\
\hline Atrial flutter & 0 & 0 & 1 & 6.3 & 0 & 0 & 0.360 \\
\hline No & 11 & 68.8 & 13 & 81.3 & 16 & 100 & 0.057 \\
\hline Total & 16 & 100 & 16 & 100 & 16 & 100 & \\
\hline
\end{tabular}

There were no statistically significant differences between groups as regard complications (Table 3).

Table (3): Drug complications

\begin{tabular}{|c|c|c|c|c|c|c|c|}
\hline \multirow[t]{2}{*}{ Complications } & \multicolumn{2}{|c|}{$\begin{array}{l}\text { Group (A) } \\
(n=16)\end{array}$} & \multicolumn{2}{|c|}{$\begin{array}{l}\text { Group (B) } \\
(n=16)\end{array}$} & \multicolumn{2}{|c|}{$\begin{array}{l}\text { Group (C) } \\
(n=16)\end{array}$} & \multirow[t]{2}{*}{$\begin{array}{l}\mathbf{P} \\
\text { Value }\end{array}$} \\
\hline & No. & $\%$ & No. & $\%$ & No. & $\%$ & \\
\hline Bradycardia & 0 & 0 & 0 & 0 & 1 & 6.3 & 0.360 \\
\hline Hypotension & 2 & 12.5 & 2 & 12.5 & 2 & 12.5 & 1 \\
\hline GIT upset & 0 & 0 & 0 & 0 & 0 & 0 & 1 \\
\hline
\end{tabular}

There was no statistically significant difference between groups as regard to Aristotle score for procedure complexity (Table 4).

Table (4): Aristotle score for procedure complexity

\begin{tabular}{|c|c|c|c|c||}
\hline $\begin{array}{c}\text { Aristotle score for } \\
\text { procedure complexity }\end{array}$ & $\begin{array}{c}\text { Group (A) } \\
(\mathbf{n = 1 6 )}\end{array}$ & $\begin{array}{c}\text { Group (B) } \\
(\mathbf{n}=\mathbf{1 6})\end{array}$ & $\begin{array}{c}\text { Group (C) } \\
(\mathbf{n}=\mathbf{1 6})\end{array}$ & P Value \\
\hline Range & $5.8-7.1$ & $5.8-7.2$ & $5.9-7.1$ & \multirow{2}{*}{0.202} \\
\hline Median & 6.30 & 6.55 & 6.70 & \\
\hline
\end{tabular}

There was statistically significant increase inotrope score in cases who received either amiodarone or magnesium sulfate as compared to placebo (Table 5). 
Table (5): Inotrope Score intraoperative

\begin{tabular}{|c|c|c|c|c||}
\hline Inotrope Score & $\begin{array}{c}\text { Group (A) } \\
(\mathbf{n = 1 6 )}\end{array}$ & $\begin{array}{c}\text { Group (B) } \\
(\mathbf{n = 1 6 )}\end{array}$ & $\begin{array}{c}\text { Group (C) } \\
(\mathbf{n = 1 6 )}\end{array}$ & P Value \\
\hline Range & $3-6$ & $5-7$ & $2-5$ & \multirow{2}{*}{$<0.001 *$} \\
\hline P1 & & $<0.001^{*}$ & $<0.001^{*}$ & \\
\hline P2 & & & $<0.001^{*}$ & \\
\hline
\end{tabular}

*: Statistically significant difference

$\mathrm{P}_{1}$ : $\mathrm{p}$ value for comparing between group $(\mathrm{A})$ and other groups

$\mathrm{P}_{2}$ : $\mathrm{p}$ value for comparing between group (B) and group $(\mathrm{C})$

There was statistically significant decrease in the heart rate in cases who received amiodarone (group c) and magnesium sulfate (group B) compared to the placebo (group A). Regarding blood pressure, there was statistically significant decrease in the blood pressure in cases who received amiodarone (group $\mathrm{C}$ ) as compared to magnesium sulfate (group B) (Table 6).

Table (6): Postoperative vital data

\begin{tabular}{|c|c|c|c|c|}
\hline $\begin{array}{c}\text { Postoperative Clinical } \\
\text { Examination }\end{array}$ & $\begin{array}{c}\text { Group (A) } \\
(\mathbf{n}=16)\end{array}$ & $\begin{array}{c}\text { Group (B) } \\
(n=16)\end{array}$ & $\begin{array}{c}\text { Group }(C) \\
(n=16)\end{array}$ & $P$ Value \\
\hline \multicolumn{5}{|l|}{ Temperature } \\
\hline Range & 37 & 37 & 37 & \multirow{2}{*}{------ } \\
\hline Median & 37 & 37 & 37 & \\
\hline \multicolumn{5}{|l|}{ Heart rate } \\
\hline Mean & 142.75 & 128.75 & 121.13 & \multirow{2}{*}{$<0.001 *$} \\
\hline S.D. & 8.087 & 7.169 & 5.714 & \\
\hline P1 & & $<0.001 *$ & $<0.001 *$ & \\
\hline P2 & & & $0.004 *$ & \\
\hline \multicolumn{5}{|l|}{$\begin{array}{l}\text { Mean Arterial } \\
\text { Pressure }\end{array}$} \\
\hline Mean \pm & 82.94 & 83.88 & 79.94 & \multirow{2}{*}{$0.038^{*}$} \\
\hline S.D. & 4.697 & 3.739 & 4.640 & \\
\hline P1 & & 0.548 & 0.059 & \\
\hline $\mathbf{P 2}$ & & & $0.015^{*}$ & \\
\hline
\end{tabular}

P: $p$ value for comparing between the three studied groups

$\mathrm{P}_{1}$ : $\mathrm{p}$ value for comparing between group (A) and other groups

$\mathrm{P}_{2}$ : $\mathrm{p}$ value for comparing between group (B) and group (C) *: Statistically significant difference

Cases who received amiodarone (group C) and magnesium sulfate (group B) show highly statistically significant increase in the postoperative inotrope score as compared to placebo (group A) (Table 7).

Table (7): Postoperative Inotrope Score

\begin{tabular}{|c|c|c|c|c||}
\hline $\begin{array}{c}\text { Postoperative } \\
\text { Inotrope Score }\end{array}$ & $\begin{array}{c}\text { Group (A) } \\
(\mathbf{n = 1 6 )}\end{array}$ & $\begin{array}{c}\text { Group (B) } \\
(\mathbf{n = 1 6 )}\end{array}$ & $\begin{array}{c}\text { Group (C) } \\
(\mathbf{n = 1 6})\end{array}$ & \multirow{2}{*}{ P Value } \\
\hline Range & $12-14$ & $11-13$ & $10-12$ & \multirow{2}{*}{$0.001^{*}$} \\
\hline Median & 13.00 & 11.00 & 11.00 & \\
\hline P1 & & $<0.001^{*}$ & $<0.001^{*}$ & \\
\hline P2 & & & 0.110 & \\
\hline
\end{tabular}

$\mathrm{p}: \mathrm{p}$ value for comparing between the three studied groups

$P_{1}$ : $p$ value for comparing between group (A) and other groups

$\mathrm{P}_{1}$ : $\mathrm{p}$ value for comparing between group (B) and group (C)

*: Statistically significant difference 


\section{DISCUSSION}

In our study we recorded total rates of intra- and postoperative arrhythmia of $16.67 \%$, SVT was the most common type encountered with an incidence of $8.33 \%$, followed by JET $6.25 \%$, the least occurring was atrial flutter $2.083 \%$. Delaney $\boldsymbol{e t}$ al. ${ }^{(14)}$ in studied early postoperative arrhythmias after pediatric cardiac surgery reported that arrhythmias occurred in 28 of the 189 patients enrolled (15\%). The most common arrhythmias were junctional ectopic tachycardia, complete atrioventricular block, ventricular tachycardia, and only one case with re-entrant supraventricular tachycardia.

The existing data in other studies reported, specific to acute postoperative arrhythmias, reports an incidence of arrhythmias ranging from $27 \%$ to $48 \%$. But in the study of Delaney et al. ${ }^{(14)}$ the arrhythmia group were younger in age, cardiopulmonary bypass time and aortic cross-clamp times were prolonged, and more complex procedure as compared to the nonarrhythmia group.

Postoperative atrial fibrillation is generally considered as a benign, self-limiting complication of cardiac surgery. However, it is associated with complications such as stroke, prolonged intensive care unit (ICU) stay and need for dialysis, leading to longer hospitalization and higher $\operatorname{costs}^{(\mathbf{1 5})}$. A possible explanation suggests that pro-arrhythmic mechanisms related to the surgery, such as inflammation, oxidative stress and sympathetic activation, reach their maximum impact at the postoperative time. Therefore, starting amiodarone immediately postoperatively, allows enough time to reach the adequate tissue levels ${ }^{(16)}$.

In the current study patients who received prophylactic amiodarone did not suffer any type of postoperative arrhythmia, as compared to the placebo group. Prophylactic magnesium sulphate was found to decrease the incidence of intra- and postoperative SVT, JET from $18.8 \%$, and $12.5 \%$ successively in placebo group to $6.3 \%$ and $6.3 \%$ successively in magnesium sulphate group. Only one case on magnesium sulphate had postoperative atrial flutter.

Cardiac surgery for congenital heart disease has changed dramatically since the first surgery in 1938. During the early era, children underwent surgery at older ages often with palliative procedures before their corrective operation. Not surprisingly, in the early era, there was considerably higher early and late mortality, including the additive risks of having more than one procedure and a long period of living with an unphysiological palliated circulation. Over time with advances in noninvasive diagnosis, surgical approach, cardiopulmonary bypass techniques, and team-based care, outcomes have improved. Children now undergo corrective surgery at a younger age and have fewer palliative procedures ${ }^{(17)}$.
The present study revealed that there was no statistically significant difference between groups regarding total surgical time and $\mathrm{CPB}$. In agreement with our findings, the study of El Amrousy et al. ${ }^{(2)}$ there was no statistically significant difference between groups regarding total surgical time and CPB.

The study on the hand demonstrated that there were no statistically significant differences between groups as regard surgical time and hospital stay. In accordance, the study of He et al. ${ }^{\left({ }^{(9)}\right.}$ reported that there was no significant difference between the $\mathrm{Mg}$ and non-Mg groups in term of CICU stay. In contrast the study of El Amrousy et al. ${ }^{(2)}$ who studied amiodarone prophylaxis versus placebo; they reported that the amiodarone group had shorter length of ICU admission as well as shorter length of hospital stay as compared to the placebo group, thus, the authors in this study claimed that amiodarone prophylaxis consequently reduced the cost of surgery but did not affect the incidence of mortality. A further study of Moak et al. ${ }^{(18)}$ was reviewed on 750 open heart surgeries for CHD they correlated the occurrence of arrhythmias with increased total surgical time, aortic cross-clamp time, cardiopulmonary bypass time.

Regarding complications in the current study, we recorded non-significant complications as bradycardia and hypotension in the magnesium and amiodarone groups versus placebo group. In agreement, the study of El Amrousy et al. ${ }^{(2)}$ reported that the efficacy of amiodarone prophylaxis was achieved without significant side effects as bradycardia and hypotension.

Survival following pediatric surgery for congenital heart disease (CHD) has improved dramatically over the past two decades; 30-day mortality estimates are now 2.5-4\%. However shortand long-term morbidity remains significant issues and hospital length of stay remains unchanged. Both are costly and are linked to poorer long-term neurodevelopmental outcomes ${ }^{(\mathbf{1 9})}$. A prospective cohort study of Murni et al. ${ }^{(20)}$ was conducted on large number of children undergoing cardiac surgery. Participants were followed up from the time of surgery until hospital discharge and 30-day mortality, and reported that 19\% patients had major complications, including cardiac arrest, need for emergency chest opening, and multiple organ failure. $12.8 \%$ died. Predictors of major complications were cyanotic congenital heart disease, longer duration of cardiopulmonary bypass and high inotropes.

In the present study we illustrated that there were no statistically significant differences between groups as regard surgical mortality. This is in agreement with the study of El Amrousy et al. ${ }^{(6)}$ in which there was no statistically significant difference between groups as regard postoperative surgical mortality. 
Evaluation of quality of care in congenital cardiac surgery is challenging. Compared to adult cardiac surgery, congenital cardiac surgery covers a smaller pediatric population and deals with many times more different diagnoses and procedures, The Aristotle Complexity Score project started in 2002, and was published in 2004. The objective of the Aristotle Score is to measure performance and to allow fair and meaningful comparison between centers and surgeons. The Aristotle Score is not specifically designed to predict mortality ${ }^{(20)}$.

In the present study we assessed the Aristotle score for procedure complexity, and found that there was no statistically significant difference between groups, this was comparable to the results in the study of He $\boldsymbol{e t ~ a l . ~}{ }^{(9)}$ regarding the distribution of surgical procedures. The distribution of surgical procedures classified by the Aristotle score was similar between the two study groups.

The inotrope score was initially described in a study by Wernovsky and Colleagues ${ }^{(21)}$. The purpose of this score was to quantify the amount of cardiovascular support received by neonates after the arterial switch operation to adjust the interpretation of measured thermodilution cardiac output based on the degree of support. This inotrope score and various adaptations have subsequently been used in clinical research as a measure of illness severity in patients undergoing CHS, despite the fact that the score has yet to be established as a predictor of outcome. In fact, there are only limited data to support the use of any clinical marker as an outcome predictor in the early postoperative period, thus hampering clinical management and high-quality research in the pediatric cardiac intensive care setting ${ }^{(22)}$.

Intraoperative inotrope score, and postoperative inotrope score revealed that there were highly statistically significant differences between groups. In the study of El Amrousy et $\boldsymbol{~ a l} .{ }^{(2)}$, they found that high doses of postoperative inotropes and hypokalemia were associated with higher incidence of JET. Hypokalemia and high-dose inotropes affect the stability of cellular membrane, leading to increase myocardial irritability and automaticity. These findings went with the results of other investigators of Delaney et al. ${ }^{(14)}$, Duke et al. ${ }^{(23)}$, and Saul et al. ${ }^{(24)}$. On the other hand, the present study revealed there was no statistically significant differences between groups as regard $\mathrm{K}$ and $\mathrm{Na}$ levels.

In the present study we assessed the participants as regard postoperative heart rate and MAP, and revealed that there were statistically significant differences between groups as regard to heart rate and mean arterial pressure postoperatively. Meanwhile, we found that there were no statistically significant differences between groups as regard arrhythmia.

Magnesium has been successfully used in the prophylaxis and treatment of a variety of cardiac arrhythmias. Magnesium is one of the more important intracellular caption's and a cofactor for many of the enzymatic reactions involved in mitochondrial energy metabolism $^{(25)}$. Despite very few studies showing a correlation between preoperative or postoperative serum hypomagnesemia and JET occurrence, magnesium has nevertheless been used successfully for prophylaxis and treatment of postoperative $\mathrm{JET}^{(\mathbf{2 6})}$. Two randomized clinical trials of Manrique $\boldsymbol{e t} \boldsymbol{a l} .{ }^{(4)}$ and Lacour-Gayet $\boldsymbol{e t}$ al. ${ }^{(27)}$ with small cohorts showed that the incidence PO of JET was significantly reduced with magnesium supplement and this effect seemed dose-related, In one of the trials with a total study population size of 99 patients, Manrique et al. ${ }^{(4)}$ found that the incidence of PO JET was reduced from 17.9 to $6.7 \%$, when $25 \mathrm{mg} / \mathrm{kg}$ of magnesium was given, and was further reduced to $0 \%$ with $50 \mathrm{mg} / \mathrm{kg}$ of magnesium.

On the other hand, we found that there were highly statistically significant differences between groups as regard to drug complications, which was more frequent among group A. In comparison to results in the study of Manrique et al. ${ }^{(4)}$, which reported that no episodes of severe bradycardia or hypotension were reported. The incidences of renal failure or cardiac dysfunction were similar in the 3 study groups. In another study done by TregglariVenzt $\boldsymbol{e t} \boldsymbol{a l} .{ }^{(28)}$ the incidences of myocardial infarction, cardiac arrest, requirement for surgical hemostasis and prolonged tracheal intubation $(>72 \mathrm{~h}$ ) were similar among the three groups. The occurrence of AF was not associated with attributable morbidity.

Finally, correlation between total surgical time and each of ICU stay and mortality was assessed in our study and found that there was no statistically significant correlation between them. Moreover, correlation between Aristotle score for procedure complexity and each of mortality, Inotrope score and postoperative HR showed that there was statistically positive significant correlation between Aristotle score for procedure complexity and Inotrope score, while correlation between Inotrope score and postoperative HR showed that there was no statistically significant correlation between them.

\section{CONCLUSIONS}

Prophylactic of either amiodarone or magnesium sulfate are safe and relatively effective in preventing early postoperative arrhythmia in children after corrective heart surgery for CHD. The exact cause of JET is not known, but several associations are identified in many studies. So further studies are recommended to help knowing risk factor that help in design proper management strategies. We recommended the usage of amiodarone or magnesium sulfate for the pediatric patient undergoing corrective surgery for CHD. 


\section{REFERENCES}

1. Peretto G, Durante A, Limite L et al. (2014): Postoperative arrhythmias after cardiac surgery: incidence, risk factors, and therapeutic management. Cardiol Res Pract., 2014:615987.

2. El Amrousy D, Elshehaby W, El Feky W et al. (2016): Safety and efficacy of prophylactic amiodarone in preventing early junctional ectopic tachycardia (JET) in children after cardiac surgery and determination of its risk factor. Pediatric Cardiology, 37(4): 734-739.

3. Edraki M, Sadeghi M, Keshavarz K et al. (2019): Early post-operative arrhythmias and their risk factors after total repair of tetralogy of Fallot in pediatric patients. International Cardiovascular Research Journal, 13(3): 96-101.

4. Manrique A, Arroyo $M$, Lin $Y$ et al. (2010): Magnesium supplementation during cardiopulmonary bypass to prevent junctional ectopic tachycardia after pediatric surgery: a randomized study. J Thorac Cardiovasc Surg., 139:162-169.

5. Tangvoraphonkchai $K$, Davenport A (2018): Magnesium and cardiovascular disease. Advances in Chronic Kidney Disease, 25(3): 251-260.

6. Manne V, Ravisekhar K (2015): Serum magnesium levels in off-pump coronary artery bypass grafting. Journal of Evolution of Medical and Dental Sciences, 4(46): 7930-7936.

7. Shahidi M, Bakhshandeh H, Rahmani K et al. (2019): Hypomagnesaemia and other electrolytes imbalances in open and closed pediatrics cardiac surgery. Pakistan Journal of Medical Sciences, 35(2): 353.

8. Fairley J, Zhang L, Glassford $N$ et al. (2017): Magnesium status and magnesium therapy in cardiac surgery: a systematic review and meta-analysis focusing on arrhythmia prevention. Journal of Critical Care, 42: 69-77.

9. He D, Aggarwal N, Zurakowski D et al. (2018): Lower risk of postoperative arrhythmias in congenital heart surgery following intraoperative administration of magnesium. The Journal of Thoracic and Cardiovascular Surgery, 156(2): 763-770.

10. Bagshaw $S$, Galbraith $P$, Mitchell $L$ et al. (2006): Prophylactic amiodarone for prevention of atrial fibrillation after cardiacsurgery: a meta-analysis. The Annals of Thoracic Surgery, 82:1927-1937

11. Buckley $M$, Nolan $P$, Slack $M$ et al. (2007): Amiodarone prophylaxis for atrial fibrillation after cardiac surgery: metaanalysis of dose response and timing of initiation. Pharmacotherapy, 27(3):360-368.

12. Esmail M, Nilufar D, Majid G et al. (2015): Prophylactic effect of amiodarone in atrial fibrillation aftercoronary artery bypass surgery; a double-blind randomized controlled clinical trial. J Cardiovasc Dis Res., 6(1):12-17.

13. Baghaei R, Arroyo M, Lin Y et al. (2010): Magnesium supplementation during cardiopulmonary bypass to prevent junctional ectopic tachycardia after pediatric cardiac surgery: A randomized controlled study. Iranian Journal of Cardiac Surgery, 139(1):162-169.
14. Delaney J, Moltedo J, Dziura J et al. (2006): Early post-operative arrhythmias after pediatric cardiac surgery. J Thorac Cardiovasc Surg., 131:1296-1300.

15. Mathew M, Fontes I, Tudor $L$ et al. (2004): A multicenter risk index for atrial fibrillation after cardiac surgery. The Journal of the American Medical Association, 291(14): 1720-1729.

16. van der does $\mathbf{W}$, de Groot $\mathbf{N}$ (2018): Prophylaxis with amiodarone for postoperative atrial fibrillation: when and who? J Thorac Dis., 10(33): 3831-3833.

17. Martin G, Jonas R (2018): Surgery for Congenital Heart Disease: Improvements in Outcomes, Am J Perinatol., 35(06): 557-560.

18. Moak J, Arias P, Kaltman J et al. (2013): Postoperative junctional ectopic tachycardia: risk factors for occurrence in the modern surgical era. Pacing Clin Electrophysiol., $36: 1156-1168$.

19. Jacobs J, He X, Mayer J et al. (2016): Mortality trends in pediatric and congenital heart surgery: an analysis of the Society of Thoracic Surgeons Congenital Heart Surgery Database. Ann Thorac Surg., 102:1345-1352.

20. Murni I, Mulyadi M, Piprim B et al. (2019): Outcome of pediatric cardiac surgery and predictors of major complication in a developing country. Ann Pediatr Cardiol., 12(1): 38-44.

21. Wernovsky G, Wypij D, Jonas R et al. (1995): Postoperative course and hemodynamic profile after the arterial switch operation in neonates and infants. A comparison of lowflow cardiopulmonary bypass and circulatory arrest. Circulation, 92:2226- 2235.

22. Gaies M, James G, Gurney A et al. (2010): Vasoactiveinotropic score as a predictor of morbidity and mortality in infants after cardiopulmonary bypass. Pediatr Crit Care Med., 11(2):234-8.

23. Duke T, Butt W, South $M$ et al. (2004): Monitoring children after cardiac surgery: a minimalist approach might be maximally effective. Crit Care Resusc., 6:306310.

24. Saul J, Scott W, Brown S et al. (2005): Intravenous amiodarone for incessant tachyarrhythmias in children a randomized, doubleblind, Antiarrhythmic Drug Trial. Circulation, 112(22):3470-3477.

25. Lee H, Ghimire S, Kim E (2013): Magnesium supplementation reduces postoperative arrhythmias after cardiopulmonary bypass in pediatrics: a meta-analysis of randomized controlled trials. Pediatr Cardiol., 34:13961403.

26. Gu W, Wu Z, Wang $P$ et al. (2012): Intravenous magnesium prevents atrial fibrillation after coronary artery bypass grafting: a meta-analysis of 7 double-blind, placebocontrolled, rand omized clinical trials. Trials, 13:41-49.

27. Lacour-Gayet F, Clarke D, Jacobs J et al. (2004): The Aristotle committee. The Aristotle score: a complexityadjusted method to evaluate surgical results. Eur J Cardiothorac Surg., 25:911-924.

28. Tregglari-Venzt $M$, Waeber $T$, Pernegerf $P$ et al. (2000): Intravenous amiodarone or magnesium sulphate is not cost-beneficial prophylaxis for atrial fibrillation after coronary artery bypass surgery. British Journal of Anaesthesia, 85(5): 690-5. 